\title{
Occurrence of extreme waves in three-dimensional mechanically generated wave fields propagating over an oblique current
}

\author{
A. Toffoli ${ }^{1,2}$, L. Cavaleri ${ }^{3}$, A. V. Babanin ${ }^{1}$, M. Benoit ${ }^{4}$, E. M. Bitner-Gregersen ${ }^{5}$, J. Monbaliu $^{2}$, M. Onorato ${ }^{6}$, \\ A. R. Osborne ${ }^{6}$, and C. T. Stansberg ${ }^{7}$ \\ ${ }^{1}$ Faculty of Engineering and Industrial Sciences, Swinburne University of Technology, P.O. Box 218, Hawthorn, \\ 3122 Vic., Australia \\ ${ }^{2}$ K.U.Leuven, Kasteelpark Arenberg 40, 3001 Heverlee, Belgium \\ ${ }^{3}$ Institute of Marine Sciences, Castello 1364/A, 30122 Venice, Italy \\ ${ }^{4}$ Saint-Venant Laboratory for Hydraulics, Université Paris-Est (joint research unit EDF R\&D, CETMEF, \\ Ecole des Ponts ParisTech), 6 quai Watier, BP 49, 78401 Chatou, France \\ ${ }^{5}$ Det Norske Veritas, Veritasveien 1, 1322 Høvik, Norway \\ ${ }^{6}$ Dipartimento di Fisica Generale, Università di Torino, Via P. Giuria 1, 10125 Torino, Italy \\ ${ }^{7}$ Marintek, P.O. Box 4125 Valentinlyst, 7450 Trondheim, Norway
}

Received: 14 September 2010 - Revised: 1 February 2011 - Accepted: 1 February 2011 - Published: 22 March 2011

\begin{abstract}
Laboratory experiments were performed to study the dynamics of three- dimensional mechanically generated waves propagating over an oblique current in partial opposition. The flow velocity varied along the mean wave direction of propagation with an increasing trend between the wave-maker and the centre of the tank. Tests with regular wave packets traversing the area of positive current gradient showed that the concurrent increase of wave steepness triggered modulational instability on otherwise stable wave trains and hence induced the development of very large amplitude waves. In random directional wave fields, the presence of the oblique current resulted in a weak reinforcement of wave instability with a subsequent increase of the probability of occurrence of extreme events. This seems to partially compensate the suppression of strongly non-Gaussian properties due to directional energy distribution.
\end{abstract}

\section{Introduction}

Extreme waves represent a serious threat for marine structures and operations (see e.g. Clauss, 2002). In this respect, an accurate description of the statistical properties of surface elevation can contribute to improving the warning

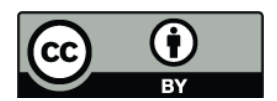

Correspondence to: A. Toffoli (toffoli.alessandro@gmail.com) criteria for extreme waves in wave forecasting and thus to enhancing safety at sea in general (Toffoli et al., 2005).

There are many mechanisms which can lead to the formation of large amplitude waves and hence to a different shape of the probability density function of the surface elevation (see Kharif and Pelinovsky, 2002, for a review). In the absence of ocean currents, numerical and theoretical works (see e.g. Onorato et al., 2001, 2005, 2006; Janssen, 2003; Babanin et al., 2007; Chalikov, 2009, among others) have demonstrated that nonlinear processes such as modulational instability have a relevant role in the formation of extreme waves, provided waves are sufficiently steep and narrow banded. Under these circumstances, large amplitude waves may occur within a fairly short scale of tens of wavelengths (Onorato et al., 2006; Janssen, 2003; Babanin et al., 2007). However, strong deviations from Gaussian statistics are only observed if waves are rather long crested, i.e., the spectral energy is concentrated over a narrow range of directions. For short crested seas (broad directional distributions), the percentage of extreme waves is notably reduced. As a result, in these circumstances, the occurrence of extreme waves does not exceed predictions from linear or second-order theory (Onorato et al., 2002; Socquet-Juglard et al., 2005; Toffoli et al., 2009). Recently, comprehensive studies on the effect of wave directional width have confirmed the existence of a transition region from strongly to weakly non-Gaussian wave fields as short crestedness is enhanced

Published by Copernicus Publications on behalf of the European Geosciences Union. 
(Onorato et al., 2009a,b; Waseda et al., 2009). This transition is determined by a balance between nonlinearity (which promotes non-Gaussian behaviour) and directional width (which suppresses non-Gaussian behaviour). Thus, if there are circumstances where the nonlinearity is locally enhanced, we can expect that non-Gaussian behaviour will persist at broader directional spreads.

In regions with strong currents, (e.g. the Gulf Stream, the Agulhas Current and the Kuroshio Current), large amplitude waves can also be expected as a result of wave-current interaction (Peregrine, 1976). Interesting in this respect, there are a number of ship accidents reported near the Agulhas Current, off the South African coast (Lavrenov, 1998; White and Fornberg, 1998). One mechanism that may lead to the formation of extreme wave events can be triggered when a random current flows with opposite or oblique direction to incident wave trains. In these circumstances, the wave energy is forced to coalesce in certain areas (White and Fornberg, 1998) with a consequent formation of large waves (caustic theory), which may result in a notable modification of the statistical distribution of wave height (Heller et al., 2008). Moreover, when waves propagate over an area of increasing adverse current (positive current gradient), the wavelength is forced to shrink with a concurrent increase of the wavenumber and wave height (see Peregrine, 1976). This results in an enhancement of the wave steepness, which is suspected to make nonlinear processes, such as the modulational instability mechanism and hence extreme waves, more likely (see, for example, Gerber, 1987; Lai et al., 1989; Stocker and Peregrine, 1999; Lavrenov and Porubov, 2006; Tamura et al., 2008). Using numerical simulation of cubic nonlinear dynamics, in this respect, Janssen and Herbers (2009) and Hjelmervik and Trulsen (2009) have tried to quantify the effect of a nonuniform current on the probability of occurrence of extreme waves. The results, however, are not conclusive and thus the role of nonlinear mechanisms on the statistical properties of the wave field, and hence the possible increase in the probability of occurrence of extreme waves, is not yet clear under these conditions.

A number of laboratory experiments have been undertaken to verify the behaviour of regular and irregular waves when opposing a strong current (see, for example, Lai et al., 1989; Chawla and Kirby, 2002; Ma et al., 2010). Experimental results, though, have mainly been obtained in wave flumes, where only long-crested waves and a collinear current (i.e., one dimensional problem) can be considered. Fewer experiments have dealt with waves crossing oblique currents (see MacIver et al., 2006) but these have been confined to a study of kinematics and linear properties. For the present investigation, we accessed the directional wave basin facility at Marintek (Norway) in order to address the more general two-dimensional problem, where a multi-directional wave field propagates obliquely over a current field in partial opposition. The aim was to verify experimentally whether a positive current gradient along the mean wave directions and the consequent current-induced increase of steepness may produce a significant effect on the modulational instability mechanism and hence on the formation of extreme waves within a wide range of wave directional spreading. In particular, we intended to ascertain the hypothesis that the wave-current interaction can compensate the suppression of strongly non-Gaussian properties in directional wave fields.

The present paper is organized as follows. In the next Section, a description of the laboratory campaign is presented. In Sect. 3, the effect of wave-current interaction on the evolution of regular wave packets is discussed to demonstrate the ability of the current to trigger modulational instability. In Sect. 4, the contribution of the wave-current interaction to the probability of occurrence of extreme waves in random directional wave fields is discussed. Concluding remarks are presented in Sect. 5.

\section{Laboratory experiments}

\subsection{Directional wave basin}

The experiments were performed in the Marintek ocean wave laboratory (Trondheim, Norway). The facility is a large rectangular wave basin with dimensions $70 \mathrm{~m} \times 50 \mathrm{~m}$. The basin is equipped with a system that is capable of changing the water depth: the bottom of the basin moves up or down in order to achieve the desired depth. The maximum depth is $h=10 \mathrm{~m}$, uniform throughout the basin. For the present experiment the water depth was fixed at $3 \mathrm{~m}$. The tank is fitted with two sets of wave-makers. Along the $50 \mathrm{~m}$ side there is a double-flap, hydraulically operated unit for generating long crested, regular and irregular waves. The second wavemaker, i.e. the one we have used for our present tests, is fitted along the $70 \mathrm{~m}$ side of the basin. It consists of altogether 144 individually computer controlled flaps. This unit can generate short crested seas within a wide range of directional distributions of the wave energy. Regular and irregular waves, with a desired angular and frequency distribution, can be generated. Also waves with the mean direction at an arbitrary angle with respect to the normal, to the wave-maker, are possible. Each of the 144 flaps is $0.434 \mathrm{~m}$ wide and hinged at a depth of $1.02 \mathrm{~m}$ below the water surface. In order to reduce wave reflections, the wave basin was equipped with two absorbing sloping beaches, which oppose the two wave-makers. Reflections in amplitude were estimated to be less than $5 \%$ after $30 \mathrm{~min}$ of irregular waves of peak period of $1 \mathrm{~s}$. The basin is also equipped with a water circulation system along the $50 \mathrm{~m}$ side, which is capable of generating a rightward current flow (see Fig. 1). A detailed description of the facility can be found in Stansberg (2008). 


\subsection{Experimental set-up}

The experiments consisted in monitoring the spatial evolution of mechanically generated regular and irregular wave fields, which were forced to traverse a current obliquely. A configuration was chosen so that the waves partially opposed the current flow. Measurements of the surface elevation were taken along the mean wave direction of propagation, where wave probes were deployed at regular intervals of $5 \mathrm{~m}$ (note that the mean wave direction is here expressed as the angle $\psi$ between the direction parallel to the wavemaker and the main direction of propagation, see Fig. 1). A number of instruments were also deployed $5 \mathrm{~m}$ apart from the central line of probes (see Fig. 1) to increase the number of observations for statistical analysis. In order to control the directional properties of the wave field, moreover, three 5probe arrays were also installed (Fig. 1). Time series were recorded at a sampling frequency of $75 \mathrm{~Hz}$. Current probes were also used to trace the current speed along the mean wave direction (i.e. across the current field). Measurements of the velocity field were gathered $0.35 \mathrm{~m}$ under the mean water level and at a sampling frequency of $75 \mathrm{~Hz}$.

Regular wave fields were generated by superimposing a monochromatic wave (carrier wave) and two side band perturbations. The carrier wave had a period of $0.8 \mathrm{~s}$ and steepness of $k a=0.1$, where $k$ is the wavenumber of the carrier wave and $a$ is its amplitude. The two perturbations were defined with amplitude $0.25 a$ and bandwidth $\Delta k / k=$ \pm 0.25 . This configuration ensured a small growth rate in the absence of the ambient current (see e.g. Janssen, 2004).

Irregular (random) waves were generated by imposing an input directional wave spectrum at the wave-maker. The energy distribution in the frequency domain was defined by a JONSWAP spectrum with peak period $T_{\mathrm{p}}=1 \mathrm{~s}$, i.e. wavelength of $1.56 \mathrm{~m}$, significant wave height $H_{\mathrm{s}}=0.08 \mathrm{~m}$ and peak enhancement factor $\gamma=6$. This configuration defines a spectral steepness $k_{\mathrm{p}} a=0.16$, where $k_{\mathrm{p}}$ is the wavenumber at the spectral peak and $a$ is half the significant wave height; the relative water depth $k_{\mathrm{p}} h=12.1$ is sufficiently large to consider waves in a deep water regime. We mention that the selected steepness is not uncommon as it was observed during a number of ship accidents reported as being due to bad weather conditions (Toffoli et al., 2005). A frequency-independent $\cos ^{N}(\vartheta)$ directional function was then applied to describe the energy in the directional domain. A number of values of the spreading coefficient $N$ were used, ranging from long to short crested conditions: respectively $N=840 ; 200 ; 90 ; 50$; and 24 . Note that these spectral configurations are identical to the ones used in Onorato et al. (2009a).

After being assigned the input spectrum, the wavemaker generates a surface elevation as an inverse Fourier transform with random amplitude and phase approximation. Therefore, the initial wave field is Gaussian (linear waves). Although there is no second-order correction of input signals,



Fig. 1. Schematic representation of the laboratory experiment.

bound waves are generated almost immediately in the basin and second-order effects are already evident after a few wavelengths from the wave-maker (see Onorato et al., 2009a, for details). We also mention that the selected spectral configurations should develop instability after about 20 25 wavelengths (cf. Socquet-Juglard et al., 2005). Hence, we expect to observe substantial deviations from Gaussian statistics in the proximity of the centre of the basin (Onorato et al., 2005, 2009a). Due to the randomn nature of the wave field, however, we cannot be sure a priori that the highest waves would occur exactly where probes were deployed.

The current was run at its maximum speed $\boldsymbol{u}=0.2 \mathrm{~m} \mathrm{~s}^{-1}$. Although the flow was normally rather stable along the current direction (cf. Stansberg, 2008), variations were observed across the current field. In this respect, the velocity was close to the nominal value in the centre of the tank, while it was reduced nearby the wave-maker and the absorption beach; the current speed along the mean wave direction is presented in Fig. 2. Note that the current field was also expected to be even slower than the observations at the first current probe right in front of the wave generator. Consequently, the wave field was subjected to a substantial positive current gradient as it propagates from the wavemaker. We also mention that initially generated waves do not have any lateral momentum, but they may obtain it while propagating across the current.

In the wave tank, the current flows in the longitudinal basin direction, so that it crosses directional wave fields generated by the multi-flap wave-maker. A specified mean wave direction was imposed on the wave field to cross the current obliquely with waves and current in partial opposition (Fig. 1). We tested two angular configurations: (a) $\psi=110 \mathrm{deg}$; (b) $\psi=120 \mathrm{deg}$. The component of the current speed along the mean wave direction measures $0.035 \mathrm{~m} \mathrm{~s}^{-1}$ and $0.068 \mathrm{~m} \mathrm{~s}^{-1}$, respectively (note that the deep-water group velocity for 1 -s waves is approximately 


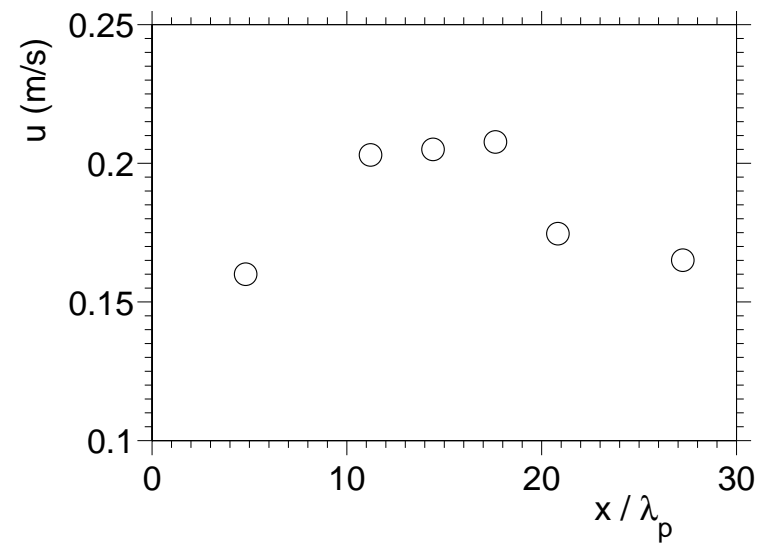

Fig. 2. Current velocity along the mean wave direction.

$0.78 \mathrm{~m} \mathrm{~s}^{-1}$ ). Observations of the significant wave height in the tank reveal that the wave amplitude increases by $7 \%$ while crossing the adverse current field; this is consistent with the fact that wave action is conserved (Peregrine, 1976). Considering the concurrent increase of wavenumber, the wave steepness is expected to enhance by $15 \%$ with respect to the nominal conditions.

It is important to note that under these circumstances, waves are forced to propagate against the side walls. This inevitably causes the formation of reflected waves, which would propagate at angles $\psi$ lower than $90 \mathrm{deg}$ and hence contaminate the measurements, especially for broad spectral conditions. To cope with this issue, 4 flaps at the furthest left-hand side of the wave-maker were deactivated in order to delay the formation of reflected waves; furthermore, probes were deployed $5 \mathrm{~m}$ downstream from the centre of the tank. A few preliminary tests on the propagation of directional wave fields $(N=24)$ were performed to address the influence of the side wall reflection at probes' location. Directional properties of the generated wave field were investigated by deriving the wave spectrum with the wavelet directional method by Donelan et al. (1996). An example of the derived directional spectrum at different distances from the wave-maker $\left(x / \lambda_{\mathrm{p}}\right)$ is presented in Fig. 3 for a mean wave direction of $120 \mathrm{deg}$. As waves propagate, part of the energy is redistributed in the frequency and directional domain by nonlinear interactions. This yields the downshift of the spectral peak and a weak broadening of the directional spectrum (cf. Toffoli et al., 2010). However, the inhomogeneity of the current flow along the mean wave direction may cause a weak refraction of the wave energy. This may partially contribute to the broadening of the directional spreading (cf. Tamura et al., 2008); it may also induce a weak variation of the mean direction of wave spectra, which was observed to slowly become increasingly current normal. Whereas the contribution of the current gradient is negligible at the first array (the directional spreading with and without current is consistent with the
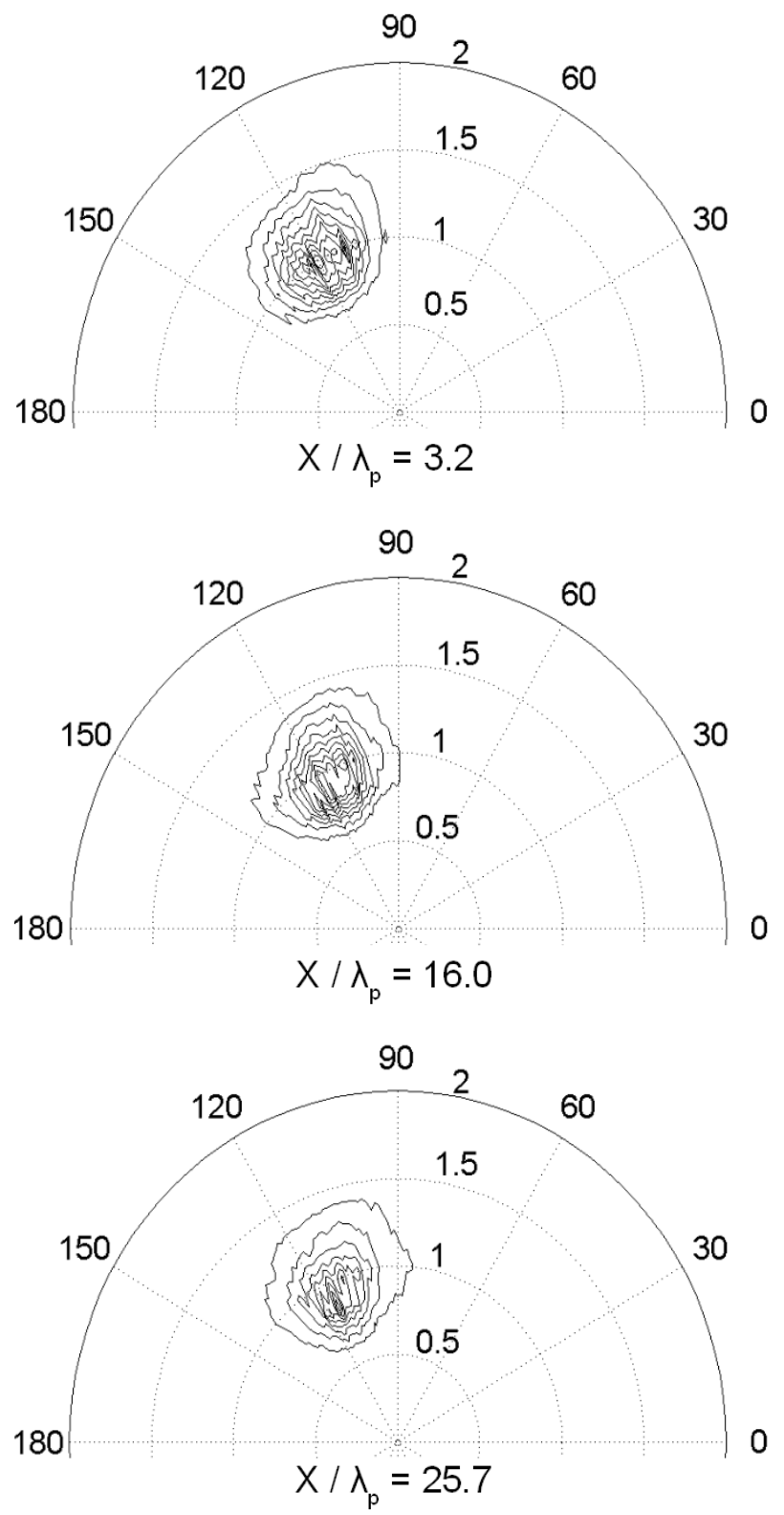

Fig. 3. Reconstructed directional wave spectra at different distances from the wave-maker $x / \lambda_{\mathrm{p}}$.

nominal condition), it becomes substantial at the last array (near the absorption beach), where the directional spreading is about $15 \%$ broader than in the absence of the current. To some extent, this does not come unexpectedly as the wave field takes some space/time to adjust to the current variation (see Hjelmervik and Trulsen, 2009). Apart from the contribution of wave-wave and wave-current interaction on the spectral shape, however, the reconstructed spectra dispel any doubt on the presence of reflected waves, as no significant energy is shown for a direction lower than $90 \mathrm{deg}$. 

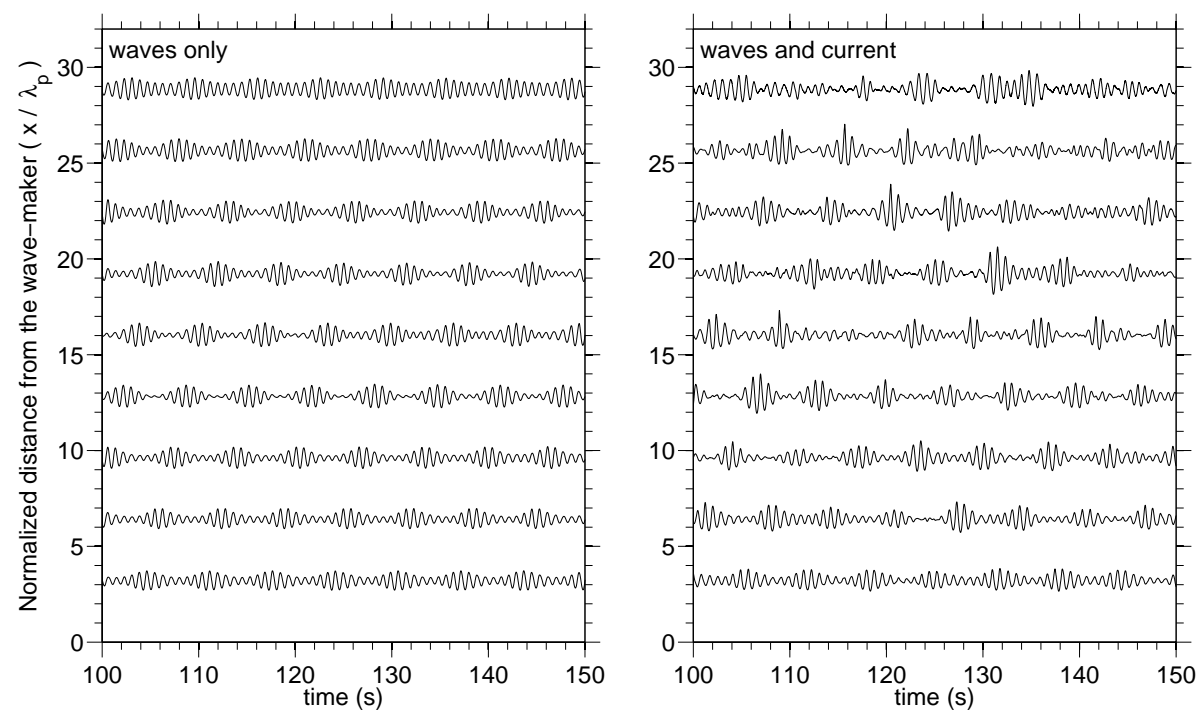

Fig. 4. Surface elevation along the tank: regular wave experiment. Regular waves are defined with a carrier wave with period of $0.8 \mathrm{~s}$ and steepness $k a=0.1$ plus two perturbations with an amplitude of $0.25 a$ and a bandwidth of $\Delta k=0.25$. The mean wave direction is 110 deg and the current speed is $0.2 \mathrm{~m} \mathrm{~s}^{-1}$.

In order to have enough waves to perform a significant statistical analysis, four realizations of the random sea surface from a given spectrum were performed by using different sets of random amplitudes and phases. For each test, 30 min of wave records were collected, including rampup time. For the present tests we have analysed the signals after $3 \mathrm{~min}$, thus having $27 \mathrm{~min}$ left for data processing.

\section{Evolution of regular wave fields}

In this section, we discuss the effect of the wave-current interaction on the evolution of regular wave packets. In Fig. 4, the surface elevation at different distances from the wave-maker is presented.

The instability growth rate was chosen to be rather small (i.e. $\approx 0.2$, see Fig. 4.3 in Janssen, 2004). Consequently, the wave packets were not able to fully develop modulational instability in the absence of a positive current gradient. Hence wave groups only tended to become slightly more accentuated along the tank, but there were no waves which could further develop into an extreme event. As shown in Fig. 5, the maximum surface elevation, i.e. the maximum wave crest, of every wave group persisted almost unaltered within the tank (note that the elevation was normalized here by the standard deviation of the water surface at the concurrent location). It cannot be excluded, nonetheless, that instability and hence extreme waves might occur if the wave field were allowed to evolve farther.

In the case when waves traverse an area of increasing current speed, on the other hand, the wavelength is forced to shorten (Peregrine, 1976) during the propagation with a

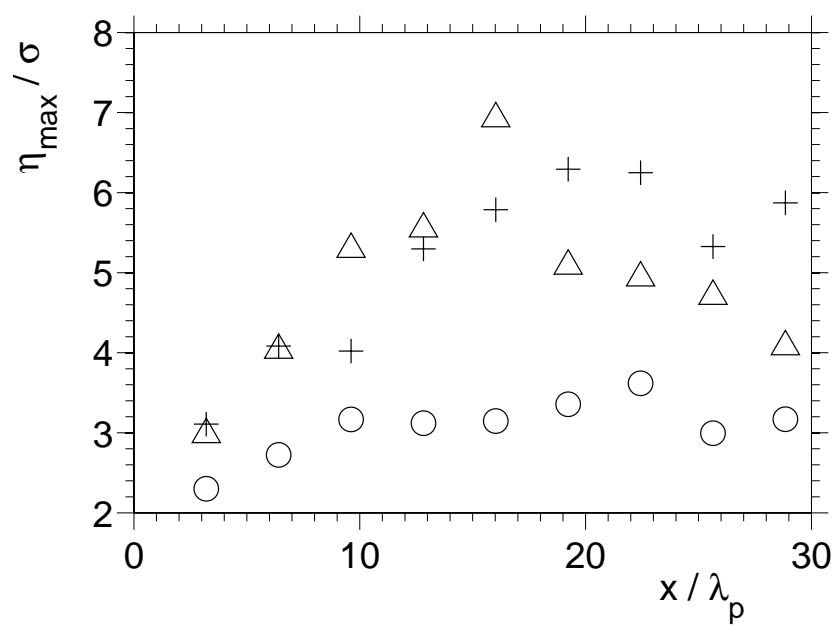

Fig. 5. Spatial evolution of the maximum surface elevation normalized by the standard deviation of the surface elevation: propagation without current (o); propagation over a current with mean wave direction of $110 \mathrm{deg}(+)$; propagation over a current with mean wave direction of $120 \mathrm{deg}(\triangle)$.

consequent increase of wave steepness. Note that we were not able to gather a direct measurement of the wavelength throughout these experiments. However, a confirmation of this enhancement was indirectly suggested by the increase of surface skewness (i.e. vertical asymmetry, see Babanin et al., 2007), which was directly related to the wave steepness according to second-order theory (cf. Tayfun, 1980). In this respect, we observed that the skewness increased on average by $15 \%$, which roughly corresponds to an equivalent increase 
of wave steepness. This resulted in a notable increase of the instability growth rate $(\approx 0.7$, in these circumstances) and thus triggered the full development of modulational instability. Although the time series appeared more erratic than the ones observed without current (see the right panel in Fig. 4), which is presumably related to the turbulence fluctuation of the current velocity, there was a robust increase of the maximum surface elevation along the tank (Fig. 5). It is interesting to mention that the maximum elevation, which is about twice the value in the initial wave packet, was reached after about 20 wavelengths for the mean wave direction of $110 \mathrm{deg}$ and 15 wavelengths for a mean wave direction of $120 \mathrm{deg}$. This outcome seems to be consistent with the fact that a stronger gradient induces a slightly larger steepness and hence faster amplitude growth. Note also that this behaviour is the one expected from the evolution of unstable wave packets (see, for example, Yuen and Lake, 1982).

After reaching its maximum, the wave elevation slightly decays towards the end of the tank. In this part of the basin, the current gradient changes sign due to the reduction of flow velocity. Because waves take some space to adjust to current variations, it is difficult to establish whether the reduction of the surface elevation is related to a natural development of modulational instability or it is otherwise forced by the effect of a negative current gradient, which tends to stretch the wavelength and hence reduces the steepness.

Repetitions of the regular wave experiment indicated that the dynamical evolution of wave packets does not change qualitatively, despite the randomness induced by the current. This tends to reinforce the hypothesis that the observed amplitude growth is only related to the current-induced increase of the wave steepness in the area of the positive current gradient.

\section{Occurrence probability of extreme waves in random wave fields}

Considering the capability of the positive current gradient to induce modulational instability and lead to the formation of large amplitude waves (see Figs. 4 and 5), it is reasonable to presume that wave-current interaction can enhance the probability of occurrence of extreme events in random (irregular) wave fields. A measure of the percentage of large waves can be conveniently expressed by the fourth order moment of the probability density function of the surface elevation, namely the kurtosis (Mori and Janssen, 2006). For linear wave fields, the statistical properties can be described by the Normal (Gaussian) probability density function, for which the kurtosis assumes the value of 3 . Traditionally, departures from the Gaussian statistics have been attributed to the presence of bound waves. In order to provide an estimate of the kurtosis, we adopt the narrowbanded approximation of the second-order theory (Longuet-
Higgins, 1963). Under such hypothesis, for deep water waves, the kurtosis can be calculated as

$\kappa_{4_{0}}=3+24\left(k_{\mathrm{p}} \sigma\right)^{2}$,

where $\sigma$ is the standard deviation of the surface elevation and $k_{\mathrm{p}}$ is the wavenumber at the spectral peak. The latter is calculated from the linear dispersion relation for waves on the surface of a current (Peregrine, 1976)

$\omega(\boldsymbol{k})=\sqrt{g \boldsymbol{k}}+\boldsymbol{k} \times \boldsymbol{u}_{\mathrm{w}}$,

where $\boldsymbol{u}_{\mathrm{w}}$ is the component of the current velocity in the mean wave direction. Note that the second-order theory has been derived from the Euler equations as a superposition of linear traveling wave solutions with random phases corrected to second-order. Nonlinear four-wave resonant and quasi-resonant interactions are not included; therefore, the spectrum does not change in time as the wave field evolves. Second-order theory usually provides a decent approximation for the kurtosis of long-crested waves. However, it tends to overpredict the value of the kurtosis for waves with directional spreading (Forristall, 2000; Toffoli et al., 2006). This is basically because in a directional spectrum the second harmonic is usually less pronounced with respect to the long crested case (see e.g. Figs. 4 and 5 in Onorato et al., 2009a).

In Fig. 6, the kurtosis is presented as a function of the distance from the wave-maker; observations and secondorder predictions are shown; for the latter, $k_{\mathrm{p}}$ and $\sigma$ are estimated from the measured time series. In the absence of the current, the spectral configuration is already sufficiently steep and narrow banded to develop modulational instability effects, provided waves are long-crested (e.g. $N=840$ ). In this respect, the kurtosis rapidly increases as waves propagate from the wave-maker and reaches a maximum of about 3.6 after 15-20 wavelengths. This strong deviation from Normality is, however, gradually suppressed for increasing directional spreading. Specifically, we observed that the kurtosis only weakly deviates from the second-order theory based predictions for $N=24$. This transition from strongly to weakly non-Gaussian properties is consistent with previous laboratory experiments in directional wave tanks (Onorato et al., 2009a; Waseda et al., 2009).

When waves traverse an area of increasing current speed, the wavelength shortens (Peregrine, 1976) with a concurrent enhancement of the wave steepness (about $14 \%$ for the present spectral configurations). For long crested conditions ( $N=840$ and 200), nonetheless, waves are already sufficiently steep to trigger instability and consequently the presence of the current does not yield any significant effect. As waves propagate, the kurtosis evolves to a maximum value that is quantitatively consistent with the one in the absence of current. When the directional spreading broadens $(N \leq 90)$, on the other hand, the kurtosis is subjected to a deceleration of its growth throughout the 


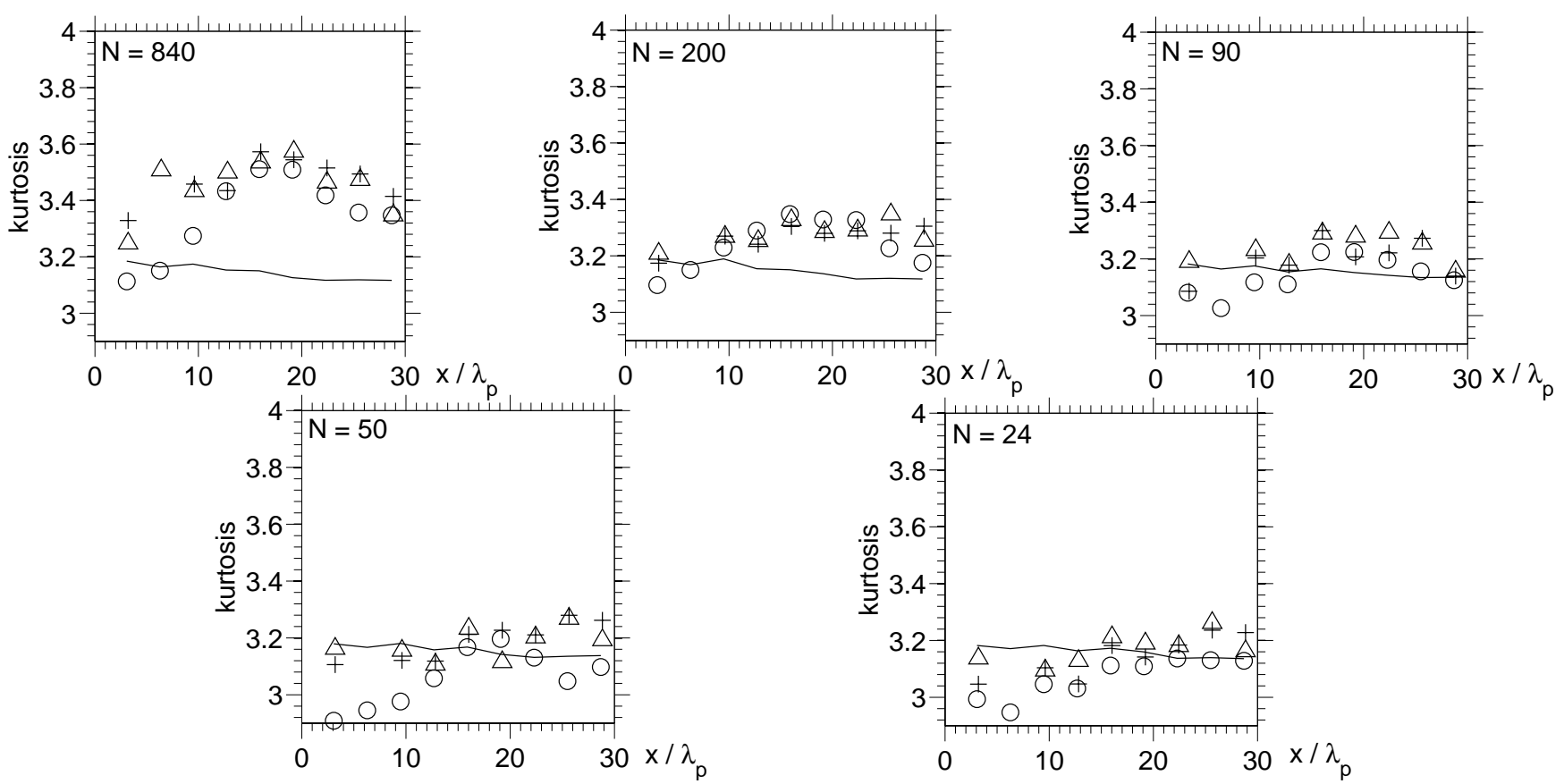

Fig. 6. Spatial evolution of the kurtosis: without current (o); with current at $110 \mathrm{deg}(+)$; with current at 120 deg $(\triangle)$; estimate based on second-order theory (continuous line).

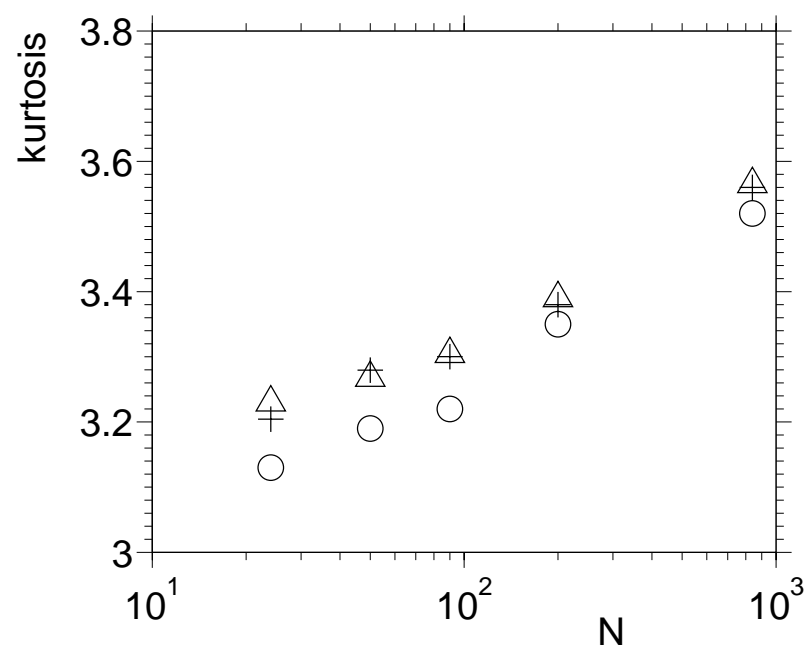

Fig. 7. Maximum kurtosis as a function of the directional spreading coefficient $N$ : without current (o); with current at $110 \mathrm{deg}(+)$; with current at $120 \mathrm{deg}(\triangle)$.

wave propagation. As such, this behaviour is similar to that observed in the absence of current. The kurtosis, however, shows the tendency to preserve slightly higher values.

In order to quantify the effect of the current on the probability of occurrence of extreme waves, it is instructive to discuss the variation of the maximum kurtosis as a function of the nominal directional spreading coefficient $N$; this is presented in Fig. 7. On the whole, a positive gradient of the current flow produces a general increase of the kurtosis. However, although extreme waves still remain less likely in short-crested wave fields rather than in long-crested conditions, the wave-current interaction sustains a slightly larger percentage of extreme events for broad directional wave fields. In this respect, the kurtosis was observed about $1 \%$ higher for $N>90$, while it was about $3 \%$ higher for $N \leq 90$. It should be noted that the difference for $N \leq 90$ is of the same order of magnitude of the statistical uncertainty i.e. \pm 0.1 as estimated with bootstrap methods. Nonetheless, the increase of kurtosis is a consistent feature for all directional spreading conditions. Considering that only a rather small degree of opposition was achieved in the tank, this outcome has to be considered qualitatively rather than quantitatively. This seems to corroborate that a current-induced increase of the wave steepness slightly compensates for the suppression of non-Gaussian behaviour due to directional width. Note that there is no substantial difference between the $110 \mathrm{deg}$ and 120 deg wave-current configurations.

\section{Conclusions}

Laboratory experiments were carried out in a directional wave tank to study the evolution of short-crested wave fields traversing an area of increasing current speed (positive current gradient). A very general condition of waves crossing obliquely and, in partial opposition, a current with changes of flow velocity along the mean wave direction was 
investigated. Two angles between waves and current of 110 and 120 deg were here discussed.

Tests in the absence of current provided consistent results with previous investigations in the same facility (Onorato et al., 2009a). Precisely, we observed that the percentage of extreme waves is substantially reduced by the broadening of the directional distribution of spectral energy. In the presence of an ambient current with a positive gradient along the mean wave direction, however, the current-induced increase of the wave steepness triggers effects related to the modulational instability of wave packets and consequently yields to the formation of large-amplitude waves.

In random wave fields, the percentage of extreme waves, which is measured by the kurtosis of the probability density function of the surface elevation, increases marginally due to the wave-current interaction. Nonetheless, it was observed that the current sustains higher kurtosis for spectral conditions with $N \leq 90$ ( $N$ is the directional spreading coefficient). This outcome seems to corroborate the hypothesis that the weak current-induced enhancement of wave steepness slightly compensates the suppression of nonGaussian behaviour due to directionality.

We mention that the incident angle between waves and current only allows a mild opposition. In view of this result, we expect that the kurtosis may increase more significantly for angles larger than $120 \mathrm{deg}$, where a stronger opposition can be accounted for.

Acknowledgements. This work has been supported by the European Community's Sixth Framework Programme through the grant to the budget of the Integrated Infrastructure Initiative HYDRALAB III within the Transnational Access Activities, Contract no. 022441. F.W.O. project G.0333.09, Australian Research Council and Woodside Energy Ltd Linkage project LP0883888 and E.U. project EXTREME SEAS (contract SCP8-GA-2009-234175) are also acknowledged.

Edited by: E. Pelinovsky

Reviewed by: two anonymous referees

\section{References}

Babanin, A., Chalikov, D., Young, I., and Savelyev, I.: Predicting the breaking onset of surface water waves, Geophys. Res. Lett., 34, L07605, doi:10.1029/2006GL029135, 2007.

Chalikov, D.: Freak waves: Their occurrence and probability, Phys. Fluids, 21, 1-4, doi:10.1063/1.3175713, 2009.

Chawla, A. and Kirby, J. T.: Monochromatic and random wave breaking at blocking points, J. Geophys. Res., 107(C7), 3067, doi:10.1029/2001JC001042, 2002.

Clauss, G.: Dramas of the sea: episodic waves and their impact on offshore structures, Appl. Ocean. Res., 24, 147-161, doi:10.1016/S0141-1187(02)00026-3, 2002.

Donelan, M. A., Drennan, W. M., and Magnusson, A. K.: Nonstationary analysis of the directional properties of propagating waves, J. Phys. Oceanogr., 26, 1901-1914, 1996.
Forristall, G.: Wave crests distributions: observations and secondorder theory, J. Phys. Oceanogr., 30, 1931-1943, 2000.

Gerber, M.: The Benjamin-Feir instability of a deep water Stokes wavepacket in the presence of a non-uniform medium, J. Fluid Mech., 176, 311-332, doi:10.1017/S0022112087000697, 1987.

Heller, E. J., Kaplan, L., and Dahlen, A.: Refraction of Gaussian seaway, J. Geophys. Res., 113, C09023, doi:10.1029/2008JC004748, 2008.

Hjelmervik, K. B. and Trulsen, K.: Freak wave statistics on collinear currents, J. Fluid Mech., 637, 267-284, doi:10.1017/S0022112009990607, 2009.

Janssen, P. A. E. M.: Nonlinear four-wave interaction and freak waves, J. Phys. Oceanogr., 33, 863-884, 2003.

Janssen, P. A. E. M.: The interaction of ocean waves and wind, Cambridge University Press, Cambridge, UK, 2004.

Janssen, T. T. and Herbers, T. H. C.: Nonlinear wave statistics in a focal zone, J. Physic. Oceanogr., 39, 1948-1964, 2009.

Kharif, C. and Pelinovsky, E.: Physical mechanisms of the rogue wave phenomenon, Eur. J. Mech. B-Fluid., 21, 561-577, doi:10.1016/j.euromechflu.2003.09.002, 2002.

Lai, R. J., Long, S. R., and Huang, N.: Laboratory studies of wave-current interaction: kinematics of the strong interaction, J. Geophys. Res., 94, 16201-16214, 1989.

Lavrenov, I.: The wave energy concentration at the Agulhas Current of South Africa, Nat. Hazard, 17, 117-127, doi:10.1023/A:1007978326982, 1998.

Lavrenov, I. and Porubov, A. V.: Three reasons for freak wave generation in the non-uniform current, Eur. J. Mech. B-Fluid., 25, 574-585, doi:10.1016/j.euromechflu.2006.02.009, 2006.

Longuet-Higgins, M.: The effect of non-linearities on statistical distribution in the theory of sea waves, J. Fluid Mech., 17, 459480, doi:10.1017/S0022112063001452, 1963.

Ma, Y., Dong, G., Perlin, M., Ma, X., Wang, G., and Xu, J.: Laboratory observations of wave evolution, modulation and blocking due to spatially varying opposing currents, J. Fluid Mech., 661, 108-129, doi:10.1017/S0022112010002880, 2010.

MacIver, R. D., Simons, R. R., and Thomas, G. P.: Gravity waves interacting with a narrow jet-like current, J. Geophys. Res., 111, C03009, doi:10.1029/2005JC003030, 2006.

Mori, N. and Janssen, P. A. E. M.: On kurtosis and occurrence probability of freak waves, J. Phys. Ocean., 36, 1471-1483, doi:10.1175/JPO2922.1, 2006.

Onorato, M., Osborne, A., Serio, M., and Bertone, S.: Freak wave in random oceanic sea states, Phys. Rev. Lett., 86, 5831-5834, doi:10.1103/PhysRevLett.86.5831, 2001.

Onorato, M., Osborne, A. R., and Serio, M.: Extreme wave events in directional, random oceanic sea states, Phys. Fluids, 14, 2528, doi:10.1063/1.1453466, 2002.

Onorato, M., Osborne, A. R., Serio, M., and Cavaleri, L.: Modulational instability and non-Gaussian statistics in experimental random water-wave trains, Phys. Fluids, 17, 078101-(1-4), doi:10.1063/1.1946769, 2005.

Onorato, M., Osborne, A., Serio, M., Cavaleri, L., Brandini, C., and Stansberg, C.: Extreme waves, modulational instability and second order theory: wave flume experiments on irregular waves, Eur. J. Mech. B-Fluid., 25, 586-601, doi:10.1016/j.euromechflu.2006.01.002, 2006.

Onorato, M., Cavaleri, L., Fouques, S., Gramstad, O., Janssen, P. A. E. M., Monbaliu, J., Osborne, A. R., Pakozdi, C., Serio, M., 
Stansberg, C., Toffoli, A., and Trulsen, K.: Statistical properties of mechanically generated surface gravity waves: a laboratory experiment in a three-dimensional wave basin, J. Fluid Mech., 627, 235-257, doi:10.1017/S002211200900603X, 2009a.

Onorato, M., Waseda, T., Toffoli, A., Cavaleri, L., Gramstad, O., Janssen, P. A. E. M., Kinoshita, T., Monbaliu, J., Mori, N., Osborne, A. R., Serio, M., Stansberg, C., Tamura, H., and Trulsen, K.: Statistical properties of directional ocean waves: the role of the modulational instability in the formation of extreme events, Phys. Rev. Lett., 102, 114502, doi:10.1103/PhysRevLett.102.114502, 2009b.

Peregrine, D. H.: Interaction of water waves and current, Adv. Appl. Mech., 9-117, 1976.

Socquet-Juglard, H., Dysthe, K., Trulsen, K., Krogstad, H., and Liu, J.: Distribution of surface gravity waves during spectral changes, J. Fluid Mech., 542, 195-216, 2005.

Stansberg, C. T.: Current effects on a moored floating platform in a sea state - OMAE2008-57621, in: Proc. 27th Int. Conf. Offshore Mechanics and Artic Engineering OMAE, Estoril, Portugal, 2008.

Stocker, J. D. and Peregrine, D. H.: The current-modified nonlinear Schrödinger equation, J. Fluid Mech., 399, 335-353, 1999.

Tamura, H., Waseda, T., Miyazawa, Y., and Komatsu, K.: Currentinduced modulation of the ocean wave spectrum and the role of nonlinear energy transfer, J. Phys. Oceanogr., 38, 2662-2684, doi:10.1175/2008JPO4000.1, 2008.
Tayfun, M. A.: Narrow-Band Nonlinear Sea Waves, J. Geophys. Res., 85(C3), 1548-1552, doi:10.1029/JC085iC03p01548, 1980.

Toffoli, A., Lefèvre, J. M., Bitner-Gregersen, E., and Monbaliu, J.: Towards the identification of warning criteria: analysis of a ship accident database, Appl. Ocean Res., 27, 281-291, doi:10.1016/j.apor.2006.03.003, 2005.

Toffoli, A., Onorato, M., and Monbaliu, J.: Wave statistics in unimodal and bimodal seas from a secondorder model, Eur. J. Mech. B-Fluid., 25, 649-661, doi:10.1016/j.euromechflu.2006.01.003, 2006.

Toffoli, A., Benoit, M., Onorato, M., and Bitner-Gregersen, E. M.: The effect of third-order nonlinearity on statistical properties of random directional waves in finite depth, Nonlin. Processes Geophys., 16, 131-139, doi:10.5194/npg-16-131-2009, 2009.

Toffoli, A., Onorato, M., Bitner-Gregersen, E. M., and Monbaliu, J.: Development of a bimodal structure in ocean wave spectra, J. Geophys. Res., 115, C03006, doi:10.1029/2009JC005495, 2010.

Waseda, T., Kinoshita, T., and Tamura, H.: Evolution of a random directional wave and freak wave occurrence, J. Phys. Oceanogr., 39, 621-639, 2009.

White, B. S. and Fornberg, B.: On the chance of freak waves at the sea, J. Fluid Mech., 255, 113-138, 1998.

Yuen, H. C. and Lake, B. M.: Nonlinear dynamics of deep-water gravity waves, Adv. Appl. Mech., 22, 20-228, 1982. 